\title{
RENORMALIZATION GROUP AND SEXTIC EFFECTIVE INTERACTION FOR THE TWO-DIMENSIONAL ISING MODEL
}

\author{
A. I. Sokolov, E. V. Orlov \\ Department of Physical Electronics, \\ Saint Petersburg Electrotechnical University, \\ 5 Professor Popov Str., St. Petersburg, 197376, Russia
}

(Received June 28, 1999)

\begin{abstract}
The universal critical value $g_{6}^{*}$ of the renormalized sextic coupling constant entering the equation of state of the two-dimensional Ising model is estimated within the renormalization-group approach. Four-loop RG expansion for $g_{6}$ is calculated and resummed by means of the Padé-Borel-Leroy technique. The estimated $g_{6}^{*}=1.10$ is obtained which agree quite well with that deduced recently by Zinn, Lai, and Fisher from the high-temperature expansions.

Key words: renormalization group, two-dimensional Ising model, free energy, equation of state, universal coupling constants.
\end{abstract}

PACS number: 05.70.Jk, 11.10.Gh, 64.60.Ak, 64.60.Fr

Although the two-dimensional (2D) Ising model in a zero magnetic field was exactly solved by L. Onsager 55 years ago, an exact analytical description of its magnetized state has not been given up to now. On the other hand, the critical behaviour of various systems in ordering fields attracts permanent attention being of prime interest both for theorists and experimentalists. Recently, the free energy (effective action) and, in particular, higher-order renormalized coupling constants $g_{2 k}$ for the basic models of phase transitions became the target of intensive theoretical studies [1-15]. These constants are related to the non-linear susceptibilities $\chi_{2 k}$, enter the scaling equation of state and thus play a very important role at criticality. Together with critical exponents and critical amplitude ratios, they are universal, i.e. possess, under $T \rightarrow T_{c}$, numerical values which are not sensitive to the physical nature of phase transition depending only on the system dimensionality and the symmetry of the order parameter.

Calculation of the universal critical values of $g_{6}, g_{8}$, etc. for three-dimensional Ising and $O(n)$-symmetric models by a number of analytical and numerical methods showed that the field-theoretical renormalization group ( $R G$ ) approach in fixed dimensions yields most accurate numerical estimates for these quantities. It is a consequence of a rapid convergence of the iteration schemes originating from renormalized perturbation theory. Indeed, the resummation of four- and five-loop RG expansions by means of the Borel-transformation-based procedures gave the values for $g_{6}^{*}$ which differ from each other by less than $0.5 \%[9,10]$ while the use of resummed three-loop RG expansion enabled one to achieve an apparent accuracy no worse than $1.6 \%[8,13]$.

The field-theoretical RG approach being very effective in $3 \mathrm{D}$ (see, e.g., Refs. [16-18,13]) is known to be powerful enough also in two dimensions. Properly resummed four-loop RG expansions lead to fair numerical estimates for critical exponents of 2D Ising model [16] and give reasonable results for its random counterpart [19]. It is natural, therefore, to use the field theory for calculation of the renormalized higher-order coupling constant $g_{6}$ of the 2D Ising model.

Within the field-theoretical language, the 2D Ising model in the critical region is described by Euclidean scalar field theory with the Hamiltonian

$$
H=\int d^{2} x\left[\frac{1}{2} m_{0}^{2} \varphi^{2}+\frac{1}{2}(\nabla \varphi)^{2}+\lambda \varphi^{4}\right]
$$

where a bare mass squared $m_{0}^{2}$ is proportional to $T$ $T_{c}^{(0)}, T_{c}^{(0)}$ being the phase transition temperature in the absence of the order parameter fluctuations. Taking fluctuations into account results in renormalizations of the mass $m_{0} \rightarrow m$, the field $\varphi=\varphi_{R} \sqrt{Z}$, and the coupling constant $\lambda=m^{2} Z_{4} Z^{-2} g_{4}$. Moreover, thermal fluctuations give rise to many-point correlations $\left\langle\varphi\left(x_{1}\right) \varphi\left(x_{2}\right) \ldots \varphi\left(x_{2 k}\right)\right\rangle$ and, correspondingly, to higherorder terms in the expansion of the free energy in powers of magnetization $M$ :

$$
\begin{aligned}
F(M)-F(0) & =m^{2}\left[\frac{1}{2} \frac{M^{2}}{Z}+g_{4}\left(\frac{M^{2}}{Z}\right)^{2}\right. \\
& \left.+\sum_{k=3}^{\infty} g_{2 k}\left(\frac{M^{2}}{Z}\right)^{k}\right] .
\end{aligned}
$$

In the critical region, where fluctuations are so strong that they completely screen out the initial (bare) interaction, the behaviour of the system becomes universal and dimensionless effective couplings $g_{2 k}$ approach their asymptotic limits $g_{2 k}^{*}$.

In order to estimate $g_{6}^{*}$ we will calculate RG expansion for $g_{6}$ and then apply Padé-Borel-Leroy resummation technique to get proper numerical results. Accurate enough numerical estimates, as is well known, may be extracted only from sufficiently long RG series. Below we'll 
find the expression for $g_{6}$ in the four-loop approximation which will be shown to provide fair numerical estimates for the quantity of interest.

The method of calculating the RG series we use here is straightforward. Since in the two dimensions higherorder bare couplings are believed to be irrelevant in the RG sense, renormalized perturbational series for $g_{6}$ can be obtained from conventional Feynman graph expansion of this quantity in terms of the only bare coupling constant $-\lambda$. In its turn, $\lambda$ may be expressed perturbatively as a function of renormalized dimensionless quartic coupling constant $g_{4}$. Substituting the corresponding power series for $\lambda$ into original expansion we can obtain the RG series for $g_{6}$. As was recently shown $[6,8,9]$ the one-, two-, three- and four-loop contributions are formed by 1, 3, 16, and 94 one-particle irreducible Feynman graphs, respectively. Their calculation gives:

$$
\begin{aligned}
g_{6} & =\frac{36}{\pi} \frac{\lambda^{3} Z^{6}}{m^{6}}\left[1-11.817855 \frac{\lambda Z^{2}}{m^{2}}+110.3727 \frac{\lambda^{2} Z^{4}}{m^{4}}\right. \\
& \left.-985.575 \frac{\lambda^{3} Z^{6}}{m^{6}}\right]
\end{aligned}
$$

The perturbational expansion for $\lambda$ emerges directly from the normalizing condition $\lambda=m^{2} Z_{4} Z^{-2} g_{4}$ and the well-known expansion for $Z_{4}[16]$ :

$$
Z_{4}=1+\frac{9}{\pi} g_{4}+5.1288114 g_{4}^{2}+10.511670 g_{4}^{3}+O\left(g_{4}^{4}\right)
$$

Combining these expressions we obtain

$$
\begin{aligned}
g_{6} & =\frac{36}{\pi} g_{4}^{3}\left(1-3.2234882 g_{4}\right. \\
& \left.+14.957539 g_{4}^{2}-85.7810 g_{4}^{3}\right) .
\end{aligned}
$$

Being a field-theoretical perturbational expansion series (5) has factorially growing coefficients, i.e. it is divergent (asymptotic). Hence, direct substitution of the fixed point value $g_{4}^{*}$ into $(5)$ would not lead to satisfactory results. To get reasonable numerical estimate for $g_{6}^{*}$ some procedure making this expansion convergent should be applied. As is well known, the Borel-Leroy transformation

$$
\begin{aligned}
& f(x)=\sum_{i=0}^{\infty} c_{i} x^{i}=\int_{0}^{\infty} t^{b} e^{-t} F(x t) d t \\
& F(y)=\sum_{i=0}^{\infty} \frac{c_{i}}{(i+b) !} y^{i} .
\end{aligned}
$$

diminishing the coefficients by the factor $(i+b)$ ! can play a role of such a procedure. Since the RG series considered turns out to be alternating the analytical continuation of the Borel transform may be then performed by using Padé approximants. With the four-loop expansion (5) in hand, we can construct three different Padé approximants: [2/1], [1/2], and [0/3]. To obtain proper approximation schemes, however, only diagonal [L/L] and near-diagonal Padé approximants should be employed [20]. That's why further we limit ourselves with approximants [2/1] and [1/2]. Moreover, the diagonal Padé approximant $[1 / 1]$ will be also dealt with although this corresponds, in fact, to the usage of the lower-order, threeloop RG approximation.

The algorithm of estimating $g_{6}^{*}$ we use here is as follows. Since the Taylor expansion for the free energy contains as coefficients the ratios $R_{2 k}=g_{2 k} / g_{4}^{k-1}$ rather than the renormalized coupling constants themselves:

$$
\begin{aligned}
F(z)-F(0) & =\frac{m^{2}}{g_{4}}\left(\frac{z^{2}}{2}+z^{4}+R_{6} z^{6}+R_{8} z^{8}+\ldots\right), \\
z^{2} & =\frac{g_{4} M^{2}}{Z}
\end{aligned}
$$

we work with the RG series for $R_{6}$. It is resummed in three different ways based on the Borel-Leroy transformation and the Padé approximants just mentioned. The Borel-Leroy integral is evaluated as a function of the parameter $b$ under $g_{4}=g_{4}^{*}$. For the fixed point coordinate the value $g_{4}^{*}=0.6125[7,21,22]$ is adopted which was extracted from lengthy high-temperature expansions and is believed to be the most accurate estimate for $g_{4}^{*}$ available nowadays. The optimal value of $b$ providing the fastest convergence of the iteration scheme is then determined. It is deduced from the condition that the Padé approximants employed should give, for $b=b_{\text {opt }}$, the values of $R_{6}^{*}$ which are as close as possible to each other. Finally, the average over three estimates for $R_{6}^{*}$ is found and claimed to be a numerical value of this universal ratio.

\begin{tabular}{|c|c|c|c|c|c|c|c|c|c|}
\hline$b$ & 0 & 1 & 1.24 & 2 & 3 & 5 & 7 & 10 & 15 \\
\hline$[1 / 1]$ & 2.741 & 2.908 & 2.937 & 3.009 & 3.077 & 3.161 & 3.212 & 3.258 & 3.301 \\
\hline$[1 / 2]$ & 2.827 & - & 2.936 & 2.877 & 2.853 & 2.828 & 2.814 & 2.800 & 2.787 \\
\hline$[2 / 1]$ & 3.270 & 2.988 & 2.936 & 2.800 & 2.667 & 2.491 & 2.380 & 2.273 & 2.171 \\
\hline
\end{tabular}

Table 1. The values of $R_{6}^{*}$ obtained by means of the Padé-Borel-Leroy technique for various $b$ within three-loop (approximant [1/1]) and four-loop (approximants [1/2] and [2/1]) RG approximations. The estimate for $b=1$ in the middle line is absent because corresponding Padé approximant turns out to be spoilt by a positive axis pole. 


\section{A. I. SOKOLOV, E. V. ORLOV}

The results of our calculations are presented in Table 1 . As one can see, for $b=1.24$ all three working approximants lead to practically identical values of $R_{6}^{*}$. Hence, we conclude that for $2 \mathrm{D}$ Ising model at criticality

$$
R_{6}^{*}=2.94, \quad g_{6}^{*}=1.10 .
$$

How close to their exact counterparts may these numbers be? To clear up this point let us discuss the sensitivity of numerical estimates given by RG expansion (5) to the type of resummation. The content of Table 1 implies that, among others, the results given by Padé approximant [2/1] turn out to be most strongly dependent on the parameter $b$. This situation resembles that for $3 \mathrm{D} O(n)$-symmetric model where Padé approximants of $[L-1 / 1]$ type for $\beta$-function and critical exponents lead to numerical estimates demonstrating appreciable variation with $b$ while for diagonal and near-diagonal approximants the dependence of the results on the shift parameter is practically absent $[13,16,18]$. In our case, Padé approximants [1/1] and [1/2] may be referred to as generating such "stable" approximations for $g_{6}^{*}$. Since for $b$ varying from 0 to 15 (i.e., for any reasonable $b$ ) the magnitude of $g_{6}^{*}$ averaged over these two approximations remains within the segment $(1.044,1.142)$ it is hardly believable that the values (8) can differ from the exact ones by more than $5 \%$.

Another way of judging the accuracy of our numerical results is based on the comparison of the values of $g_{6}^{*}$ given by four subsequent RG approximations available. While within the one-loop order we get $g_{6}^{*}=2.633$ which is obviously a very bad estimate, the taking into account of higher-order RG contributions to $g_{6}$ improve the situation markedly. Indeed, two-, three-, and fourloop RG series when resummed by means of the PadéBorel technique with the use of "most stable" approximants $[0 / 1],[1 / 1]$, and $[1 / 2]$ yield for $g_{6}^{*}$ the values 0.981 , 1.129 , and 1.051 , respectively. Since this set of numbers demonstrates an oscillatory convergence one may expect that the exact value of renormalized sextic coupling constant lies between the higher-order - three-loop and four-loop - estimates. It means that the deviation of numbers (8) from the exact values would not exceed $5 \%$.

Thus, we see that the four-loop RG expansion for $g_{6}$ and elaborated approximation scheme lead to sufficiently accurate numerical data. On the other hand, the above arguments, as always when we deal with diverging series, should be interpreted as suggestive, i.e. they would help us to fix only an apparent accuracy. It is of prime importance therefore to compare our estimates with those obtained by other methods. Recently, S.-Y. Zinn, S.-N. Lai, and M. E. Fisher analyzing high temperature series for various $2 \mathrm{D}$ Ising lattices found that $R_{6}^{*}=2.943 \pm 0.007$ [7]; an almost identical value was obtained later in Ref. [14]. Our result for $R_{6}^{*}$ is seen to be in brilliant agreement with this number. Of course, practical coincidence of the lattice and four-loop RG estimates is occasional and cannot be considered as a manifestation of extremely high accuracy of the methods discussed. The closeness of these estimates to each another, however, unambiguously demonstrates high power of both the approaches. Moreover, such a closeness sheds light on the role of a singular contribution to $g_{6}$ which cannot be found perturbatively: this contribution is seen to be numerically small.

It is instructive also to compare our results with those given by another field-theoretical approach - the famous $\epsilon$-expansion. Today for the Ising systems only three terms in the $\epsilon$-expansion for $R_{6}$ are known [23]:

$$
R_{6}^{*}=2 \epsilon\left(1-\frac{10}{27} \epsilon+0.63795 \epsilon^{2}\right)
$$

Let us apply a simple Padé-Borel procedure to this series as a whole and to the series in brackets and then put $\epsilon=2$. We find $R_{6}^{*}=3.19$ and $R_{6}^{*}=3.12$ respectively, i.e. the numbers which differ from our estimate by less than $9 \%$. Keeping in mind lack of a small parameter these values of $R_{6}^{*}$ may be referred to as consistent. Proper account for higher-order terms in the $\epsilon$-expansion for $R_{6}$ should make corresponding numerical estimates closer to those extracted from 2D RG and high-temperature series. Very good agreement between the first number (8) and the estimate $R_{6}^{*}=2.95 \pm 0.03$ [14] obtained recently by matching the $\epsilon$-expansion available with the exact results known for $D=1$ and $D=0$ may be considered as an argument in favour of this belief.

It is a great honour to devote this paper to the memory of Academician N. N. Bogoliubov. The work was supported by the Ministry of General and Professional Education of Russia under Grant No. 97-14.2-16.
[1] C. Bagnuls, C. Bervillier, Phys. Rev. B 41, 402 (1990).

[2] C. M. Bender, S. Boettcher, L. Lipatov, Phys. Rev. Lett. 68, 3674 (1992); C. M. Bender, S. Boettcher, Phys. Rev. D 48, 4919 (1993); 51, 1875 (1995).

[3] N. Tetradis, C. Wetterich, Nucl. Phys. B 422, 541 (1994).

[4] M. M. Tsypin, Phys. Rev. Lett. 73, 2015 (1994); Phys. Rev. B 55, 8911 (1997).
[5] T. Reisz, Phys. Lett. B 360, 77 (1995).

[6] A. I. Sokolov, Fiz. Tverd. Tela 38, 640 (1996) [Sov. Phys. Solid State 38, 354 (1996)].

[7] S.-Y. Zinn, S.-N. Lai, M. E. Fisher, Phys. Rev. E 54, 1176 (1996).

[8] A. I. Sokolov, V. A. Ul'kov, E. V.Orlov, J. Phys. Stud. 1, 362 (1997).

[9] A. I. Sokolov, E. V. Orlov, V. A.Ul'kov, Phys. Lett. A 
227, 255 (1997).

[10] R. Guida, J. Zinn-Justin, Nucl. Phys. B 489, 626 (1997).

[11] T. R. Morris, Nucl. Phys. B 495, 477 (1997).

[12] P. Butera, M. Comi, Phys. Rev. E 55, 6391 (1997).

[13] A. I. Sokolov, Fiz. Tverd. Tela 40, 1284 (1998) [Sov. Phys. Solid State 40, 1169 (1998)].

[14] A. Pelissetto, E. Vicari, Nucl. Phys. B 522, 605 (1998).

[15] A. I. Sokolov, E. V. Orlov, V. A. Ul'kov, S. S. Kashtanov, Phys. Rev. E 60, 1344 (1999), in press; Preprint hep-th/9810082.

[16] G. A. Baker, B. G. Nickel, D. I. Meiron, Phys. Rev. B 17, 1365 (1978).
[17] J. C. Le Guillou, J. Zinn-Justin, Phys. Rev. B 21, 3976 (1980).

[18] S. A. Antonenko, A. I. Sokolov, Phys. Rev. E 51, 1894 (1995).

[19] I. O. Mayer, A. I. Sokolov, B. N. Shalayev, Ferroelectrics 95, 93 (1989); I. O. Mayer, J. Phys. A 22, 2815 (1989).

[20] G. A. Baker, Jr. and P. Graves-Morris. Padé Approximants (Addison-Wesley, Reading, MA, 1981).

[21] G. A. Baker, Jr., Phys. Rev. B 15, 1552 (1977).

[22] P. Butera, M. Comi, Phys. Rev. B 54, 15828 (1996).

[23] J. Zinn-Justin, Quantum Field Theory and Critical Phenomena (Clarendon Press, Oxford, 1996).

\title{
РЕНОРМГРУПА ТА ШЕСТІРНА ЕФЕКТИВНА ВЗАЄМОДІЯ ДЛЯ ДВОВИМІРНОЇ МОДЕЛІ ІЗИНГА
}

\author{
А. І. Соколов, С. В. Орлов \\ Санкт-Петербургсъкий електротехнічний університет, кафедра фізичной електроніки, \\ вул. проф. Попова, 5, Санкт-Петербург, 197376, Росія
}

\footnotetext{
Універсальне критичне значення $g_{6}^{*}$ ренормалізованої константи шестірної взаємодії, що входить у рівняння стану двовимірної моделі Ізинга, ощінюється в рамках ренормгрупового підходу. Чотирипетлеве ренормгрупове розвинення для $g_{6}$ обчислене і пересумоване за методом Паде-Бореля-Леруа. Отримане значення $g_{6}^{*}=1.10$ добре узгоджується зі значенням, яке нещодавно визначили Цінн, Лай та Фішер з високотемпературних розвинень.
} 